\title{
Mechanokinetics of receptor-ligand interactions in cell adhesion
}

\author{
Ning Lii ${ }^{1} \cdot$ Shouqin L $\ddot{u ̈}^{1} \cdot$ Yan Zhang ${ }^{1} \cdot$ Mian Long $^{1}$
}

Received: 20 November 2014 / Revised: 18 December 2014 / Accepted: 12 January 2015 / Published online: 14 May 2015

(C) The Chinese Society of Theoretical and Applied Mechanics; Institute of Mechanics, Chinese Academy of Sciences and Springer-Verlag Berlin Heidelberg 2015

\begin{abstract}
Receptor-ligand interactions in blood flow are crucial to initiate such biological processes as inflammatory cascade, platelet thrombosis, as well as tumor metastasis. To mediate cell adhesion, the interacting receptors and ligands must be anchored onto two apposing surfaces of two cells or a cell and a substratum, i.e., two-dimensional (2D) binding, which is different from the binding of a soluble ligand in fluid phase to a receptor, i.e., three-dimensional (3D) binding. While numerous works have been focused on 3D kinetics of receptor-ligand interactions in the immune system, 2D kinetics and its regulations have been less understood, since no theoretical framework or experimental assays were established until 1993. Not only does the molecular structure dominate 2D binding kinetics, but the shear force in blood flow also regulates cell adhesion mediated by interacting receptors and ligands. Here, we provide an overview of current progress in $2 \mathrm{D}$ binding and regulations, mainly from our group. Relevant issues of theoretical frameworks, experimental measurements, kinetic rates and binding affinities, and force regulations are discussed.
\end{abstract}

Keywords Receptor-ligand interactions - Selectins · $\beta_{2}$ integrins $\cdot 2 \mathrm{D}$ binding kinetics

Mian Long

mlong@imech.ac.cn

1 Key Laboratory of Microgravity (National Microgravity Laboratory), Center of Biomechanics and Bioengineering, and Beijing Key Laboratory of Engineered Construction and Mechanobiology, Institute of Mechanics, Chinese Academy of Sciences, Beijing 100190, China

\section{Introduction}

Cell adhesion is a fundamental biological process that is mediated by specific interactions between adhesion receptors and their ligands on other cell surfaces or in the extracellular matrix $[1,2]$. Such adhesions are important to processes such as inflammatory cascade, platelet thrombosis, as well as tumor metastasis [3]. As a first example, the interactions between selectins and glycoconjugates mediate neutrophil (PMN) tethering to and rolling on vascular surfaces at sites of inflammation or injury [4-6] (Fig. 1). The selectin family of adhesion molecules has three known members: P-, E-, and $\mathrm{L}$-selectin. Their common structure is an $\mathrm{N}$-terminal, C-type lectin (Lec) domain, followed by an epidermal growth factor (EGF)-like module, multiple copies of consensus repeat (CR) units characteristic of complement binding proteins, a transmembrane segment, and a short cytoplasmic domain [6]. Pselectin glycoprotein ligand 1 (PSGL-1), as a major selectin ligand, consists of a homodimer cross-linked by disulfate bonds and binds to selectins by its N-terminal peptide, which includes three tyrosine sulfates and the core-2 O-glycan [7-11]. As a second example, the interactions between $\beta_{2}$ integrin of lymphocyte function-associated antigen-1 (LFA1) or macrophage-1 antigen (Mac-1), expressed on PMNs, and intercellular adhesion molecule 1 (ICAM-1), expressed on endothelial cells, dominate the slow rolling, firm adhesion, and intravascular crawling of PMNs under blood flow [12-14] (Fig. 1). LFA-1 and Mac-1 share a common $\beta_{2}$ subunit non-covalently associated with a respective $\alpha$ subunit. The $\beta_{2}$ subunit is composed of an I-like domain, a hybrid domain, a plexin/semaphorin/integrin domain, four integrin epidermal growth factor-like domains, a transmembrane domain, and a cytoplasmic tail, while the $\alpha$ subunit includes an I-domain, a $\beta$-propeller, a thigh domain, two 


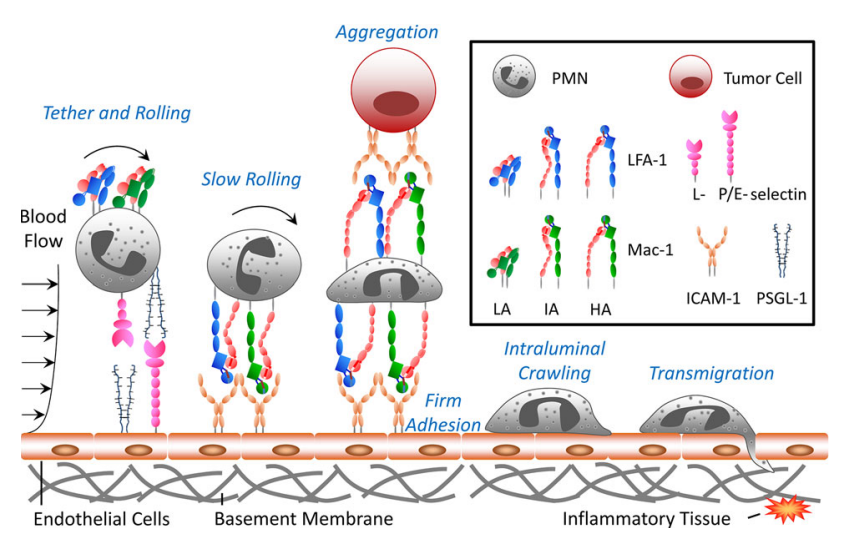

Fig. 1 Multistep process of leukocyte recruitment under blood flow in inflammation. A neutrophil undergoes capture and rolling (or tethering) on the endothelium through selectin-PSGL-1 bonds, followed by slow rolling and firm adhesion through the $\beta_{2}$-integrins LFA- 1 and Mac-1 as well as intraluminal crawling and transmigration through the endothelium to the inflamed tissue. Interactions between $\beta_{2}$-integrin on PMNs and ICAM-1 on tumor cells initiate binding within the local tumor microenvironment in blood flow

calf domains, a transmembrane domain, and a cytoplasmic tail $[15,16]$. Inside-out signaling triggered by selectins and chemokines, or outside-in signaling triggered by ligand binding, induces $\beta_{2}$ integrins to undergo a dramatic transition from bent low-affinity (LA) to extended intermediate(IA) or high-affinity (HA) conformation [17-20]. ICAM-1, a member of the super IgG family, consists of five IgG-like domains (D1-D5) and binds to Mac-1 via the D3 domain and LFA-1 via the D1 domain, respectively [21,22]. As a third example, circulating immunoglobulin $\mathrm{G}$ ( $\mathrm{IgG}$ ) binds to foreign particles or damaged tissue through dual antigenbinding fragments $(\mathrm{Fab})$. Their conserved $\mathrm{Fc}$ fragment is available for binding to $\mathrm{Fc} \gamma$ receptors ( $\mathrm{Fc} \gamma \mathrm{Rs}$ ) on the immune cell surface, which triggers a wide variety of immune responses [23,24].

Interactions of cellular adhesive molecules are determined by their intrinsic kinetics (reaction rates and binding affinity), since kinetic parameters govern how likely and how fast the adhesion occurs, and how strong and how long the bond remains bound. In a typical 2D binding, at least two specific aspects arise as compared to 3D binding [25]. One is the coupling of kinetics and mechanics, so-called mechanochemical coupling, of receptor-ligand interactions. In blood flow, cell adhesion is regulated by hemodynamic forces which are translated into external forces on interacting molecules. Bond formation and dissociation of interacting adhesive molecules provide the physical linkages between cells. Not only does applied force regulate the lifetime of molecular interactions, just as pressure affects the chemical rates [26,27], but the force loading rate also affects the bond strength of molecular interactions [28,29]. For example, the formation of immunological synapse between a T-cell and an antigen-presenting cell is governed by both $2 \mathrm{D}$ binding kinetics of interacting molecule pairs (i.e., T-cell receptor vs. major histocompatibility molecules, integrin vs. ICAM-1 ligand) and mechanics of the cell membrane (i.e., stiffness, rigidity) [30].

Another aspect is the stochastic nature of receptor-ligand interactions. In contrast to 3D binding, where thousands of interacting receptors and ligands are involved and molecular fluctuation of individual molecules is averaged out by their statistical behaviors, molecular bonds in 2D binding are rarely formed inside the contact area. This infrequent occurrence of adhesion introduces the stochastic nature of individual molecules. For example, there are only single or a few bonds of P-selectin-ligand bonds involved in leukocyteendothelium interactions [31]. Moreover, the number of bonds varies each time. Taken together, these aspects suggest that new theoretical models and experimental measurements are required to understand quantitatively $2 \mathrm{D}$ kinetics of receptor-ligand interactions.

In this review, we provided an overview of the current progress in the quantitative understanding of $2 \mathrm{D}$ kinetics and forced dissociation of receptor-ligand bindings. Four issues were discussed, including theoretical bases, experimental measurements, 2D kinetics and affinities, and force regulations. These summaries provide new insights into understanding the receptor-ligand interactions in immune responses.

\section{Theoretical framework for receptor-ligand kinetics}

Consider a second-order forward and first-order reverse reaction,

$R+L \underset{k_{\mathrm{r}}}{\stackrel{k_{\mathrm{f}}}{\rightleftarrows}} B$,

where $R, L$, and $B$ denotes, respectively, the receptor, ligand, and bond. In 3D binding, kinetics of a soluble ligand binding to a receptor follows a simple, deterministic kinetic equation,

$\mathrm{d}[B] / \mathrm{d} t=k_{\mathrm{f}}[R][L]-k_{\mathrm{r}}[B]$,

where $[R],[L]$, and $[B]$ denote the concentrations of receptor, ligand, and bond, respectively (in units of molar concentration or $\mathrm{M}$ ), and $k_{\mathrm{f}}$ (in unit of $\mathrm{M}^{-1} \mathrm{~s}^{-1}$ ) and $k_{r}$ (in units of $\mathrm{s}^{-1}$ ) are the forward and reverse rates, respectively. $K_{\mathrm{a}}\left(=k_{\mathrm{f}} / k_{\mathrm{r}}\right)$ is the binding affinity (in units of $\mathrm{M}^{-1}$ ) when the reaction reaches an equilibrium state.

Two-dimensional binding of receptor-ligand interactions in cell-cell or cell-substrate adhesions is a stochastic process regulated by applied forces. On one hand, the stochastic nature of such a binding can be described using a probabilis- 
tic model. The basic idea is to define the probability of bonds, instead of the concentration of bonds, since the adhesion is no longer a deterministic process. A probabilistic modeling was developed based upon a small system kinetics first proposed by McQuarrie [32], and the adhesion probability, $P_{\mathrm{a}}$, at contact time $t$ follows [33-36],

$P_{\mathrm{a}}=1-\exp \left\{-A_{\mathrm{c}} m_{\mathrm{r}} m_{1} K_{\mathrm{a}}^{0}\left[1-\exp \left(-k_{\mathrm{r}}^{0} t\right)\right]\right\}$,

where $K_{\mathrm{a}}^{0}$ (in units of $\mu \mathrm{m}^{2}$ ) and $k_{\mathrm{r}}^{0}$ are, respectively, the zero-force binding affinity and reverse rate, $m_{\mathrm{r}}$ and $m_{1}$ are, respectively, the site densities of receptor and ligand (in units of $\mu \mathrm{m}^{-2}$ ), and $A_{\mathrm{c}}$ is the contact area (in units of $\mu \mathrm{m}^{2}$ ). Twodimensional kinetics parameters of $A_{\mathrm{c}} m_{1} K_{\mathrm{a}}^{0}$ (if $m_{\mathrm{r}}$ is known) or $A_{\mathrm{c}} K_{\mathrm{a}}^{0}$ (if both $m_{\mathrm{r}}$ and $m_{1}$ are known) and $k_{\mathrm{r}}^{0}$ can be predicted by fitting the experimental measurements of binding curves ( $P_{\mathrm{a}} \sim t$ curves) to the model (Eq. 3), and 2D forward rate $k_{\mathrm{f}}$ (in units of $\mu \mathrm{m}^{2} \mathrm{~s}^{-1}$ ) can be obtained by the definition $\left(=K_{\mathrm{a}}^{0} \times k_{\mathrm{r}}^{0}\right)$. Note that non-specific binding should be subtracted out of total binding before fitting the curve using Eq. 3 .

On the other hand, force regulates the formation and dissociation of bonds in blood flow. Two parameters are used to quantify the effect: one is the bond rupture force or bond strength, and another is bond lifetime. The bond rupture force depends on the rate of force application or force loading rate [28,29,37-42], and other extrinsic physical parameters [43]. Bond lifetime is governed by external forces, as proposed by Bell [27] and Dembo [26],

$k_{\mathrm{r}}=k_{\mathrm{r}}^{0} \exp \left( \pm \frac{a f}{k_{\mathrm{B}} T}\right)$

where $k_{\mathrm{r}}$ is the reverse rate at force $f, a$ is the interaction range, and $k_{\mathrm{B}}$ and $T$ are, respectively, the Boltzmann constant and absolute temperature. Noting that the bond lifetime, $\tau$, is the reciprocal of the reverse rate $\left(\tau=1 / k_{\mathrm{r}}\right)$ at any given force, $f$, Eq. 4 results in two mechanisms of forced dissociation of bonds: bond lifetime $\tau$ decreases with $f$ (if Eq. 4 is positive), which is termed slip bond $[27,44,45]$, or increases with $f$ (if Eq. 4 is negative), which is termed catch bond [26,44-48]. Experimental measurements of bond lifetime at systematically varied forces can be used to determine the force dependence of bond lifetime, which is used to test the theoretical predictions (Eq. 4).

It should be noted that the 2D binding affinity could also be theoretically converted from 3D binding affinity through structural analyses and multiscale (atomic-scale molecular dynamics, Monte-Carlo, and lattice) simulations [49], although this review does not focus on the proteins of interest reported here.

\section{Experimental approaches for quantifying 2D kinetics and forced dissociation}

Until 1993, 2D kinetics measurements were not available experimentally [50]. From then on, many experimental assays have been developed by coordinating the biological experiments and mechanical measurements. These include micropipette aspiration $[25,31,36,51]$, optical tweezers [37, 52,53], biological force probes [45,54-56], atomic force microscopy (AFM) [38,39,43,57-61], flow chamber [6265], microcantilever needle [28], centrifugation [66], rosetting [67], cone-plate viscometer [68,69], surface force apparatus [70,71], and fluorescence recovery after photobleaching (FRAP) [72,73]. Here, two assays of micropipette aspiration and atomic force microscopy are exemplified to demonstrate how they work.

In a micropipette aspiration assay, two cells (generally a human red blood cell (RBC) and a nucleated cell, respectively, expressing or coated with a receptor and the counterpart ligand) are aspirated by two micropipettes with diameters of $\sim 1.5-3 \mu \mathrm{m}$ via a suction pressure of $1-4$ $\mathrm{mmH}_{2} \mathrm{O}[31,36,51,74]$ (Fig. 2a). Adhesion between the RBC and the nucleated cell is staged by placing them in controlled contact via micromanipulation (Fig. 2b). The presence of adhesion and the adhesion force at the end of a given contact period are detected mechanically by observing microscopically the deflection of the flexible RBC membrane upon retracting it away from the nucleated cell (Fig. 2c, d). This contact-retraction cycle is repeated 100 times to estimate the adhesion probability, $P_{\mathrm{a}}$, at that contact duration, $t$. About 100 pairs of cells are used to obtain several $P_{\mathrm{a}}$ vs. $t$ curves that correspond to different receptor and ligand densities, $m_{\mathrm{r}}$ and $m_{1}$. Equation 3 is used to estimate the zero-force reverse rate, $k_{\mathrm{r}}^{0}$ and effective binding affinity, $A_{\mathrm{c}} m_{1} K_{\mathrm{a}}^{0}$ (if $m_{\mathrm{r}}$ was known) or $A_{\mathrm{c}} K_{\mathrm{a}}^{0}$ (if both $m_{\mathrm{r}}$ and $m_{1}$ were known).

In an atomic force microscopy assay, a receptor or ligand is coated directly or captured via a capturing monoclonal antibody ( $\mathrm{mAb}$ ) onto the AFM cantilever tip. Purified counterpart ligands or receptors are incorporated in lipid vesicles and

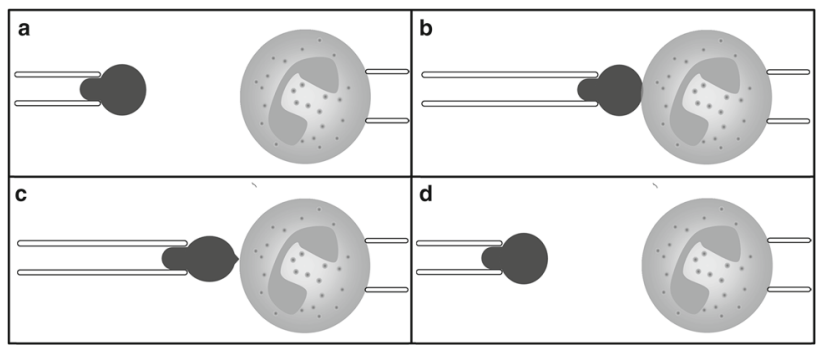

Fig. 2 A test cycle of micropipette aspiration assay in four phases of approach (a), contact (b), withdrawal (c), and detachment (d). Here, a red blood cell (dark cell) serves as a force transducer to determine an adhesive event on membrane deflection 


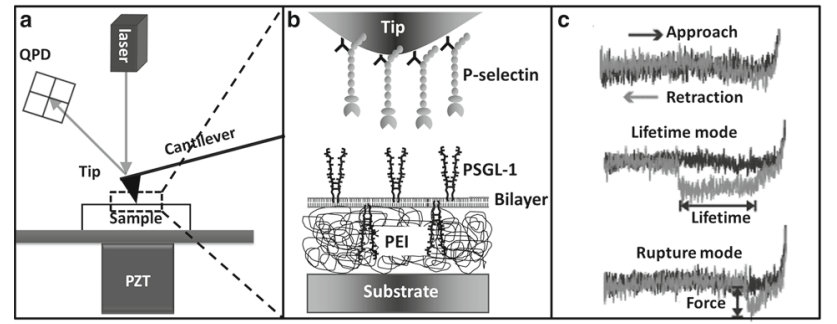

Fig. 3 An atomic microscopy (AFM) approach in molecular biomechanics. a Schematic of the AFM instrument. b Functionalizing the AFM tip using capture $\mathrm{mAbs}$ or PEI polymer cushion to orient receptors or ligands properly. $\mathbf{c}$ Force-displacement curves illustrating two working modes of bond lifetime and rupture force

then reconstituted by vesicle fusion in a polyethylenimine (PEI) polymer-supported lipid bilayer onto a mica or glass surface before use (Fig. 3b) [38,39,43,57-61]. The ligandor receptor-reconstituted lipid bilayer is placed on the AFM stage, which is repeatedly driven by a piezoelectric translator (PZT) to approach the receptor- or ligand-coated cantilever tip, to make contact to allow reversible bond formation and dissociation, and to retract away to allow observation of the adhesion event and measurement of lifetime or rupture force, if any (Fig. 3c). Adhesion lifetime or rupture force for each approach-contact-retract cycle is collected from a quad photodetector (QPD) (Fig. 3a). Different locations on each lipid bilayer are tested for 150-400 cycles at each location to collect a set of adhesion events and lifetimes or rupture forces, and all experiments are repeated using a set of different lipid bilayers. Measured $P_{\mathrm{a}}$ vs. $t$ data is fitted to the model (Eq. 3) to obtain the kinetic parameters $\left(k_{\mathrm{r}}^{0}\right.$ and $\left.A_{\mathrm{c}} m_{\mathrm{r}} m_{1} K_{\mathrm{a}}^{0}\right)$ [43]. Bond lifetime data, $<\tau>$ vs. $f$, are measured to test the forced dissociation hypotheses (Eq. 4) [44], and bond rupture forces are measured at given force loading rates $[39,43,58]$.

It should be pointed out that slight differences might exist in determining quantitatively kinetic parameters, bond rupture force, and bond lifetime when different assays are used for the same molecular system (Table 1). This should not be a surprise since experimental conditions are hard to be kept identical from one assay to another. Nevertheless, these assays provide a new insight into quantifying the binding kinetics and force dependence of dissociation of receptorligand interactions.

Recently, fluorescence resonance energy transfer (FRET) is also used to measure the 2D kinetics of TCR-pMHC binding and tension across vinculin in stable focal adhesions $[75,76]$. As a fluorescence-based assay, FRET is powerful for defining the binding kinetics of protein-protein interactions where a fluorescent donor in its excited state transfers the emitted energy non-radioactively to a fluorescent acceptor within 1-10 nm distance [77]. These FRET signals provide a high degree of spatial and temporal sensitivity, which are suitable for real-time measurement of protein conformational change and intracellular signal transduction [75-78]. Nevertheless, this fluorescence-based assay is more complicated than those mechanical-based ones discussed above, and also asks for FRET sensor design, fluorophore conjugation, and additional force sensor calibration. To our knowledge, there are few FRET works in the field of 2D selectin/integrinligands binding so far. It is expected that the data collected from both assays will be compared in the future.

\section{Progress update of $2 \mathrm{D}$ receptor-ligand binding and forced dissociation}

\subsection{Modeling of cell aggregation and cell adhesion mediated by receptor-ligand interactions}

Under physiological blood flow, blood cells not only form homotypic or heterotypic aggregates, they are also able to adhere to the endothelium. Since aggregation and adhesion between two cells are first driven to make contact by shear flow and then are cross-linked by underlying receptor-ligand bonds, a two-body collision theory is coupled with a probabilistic model of small system kinetics and a mechanochemical coupling theory of forced bond dissociation. We first proposed a theoretical model to predict the shear-induced formation and break-up of doublets in three sequential phases: (1) formation upon two-body collision between singlets; (2) evolution of bonds at low shear rate; and (3) break-up of bonds at high shear rate. The predictions upon the model are found to be in good agreement with measurements and enable us to estimate the binding affinity and kinetic rates for three types of doublets cross-linked by two receptor-ligand systems [69]. Then the model was modified by adding a term of time-dependent forward rate to quantify the aggregation of PMNs and tumor cells [68]. Next, the model was further developed to account for the transition from zero-bond singlet to $n$-bond doublet by adding a term of first bond forming probability from those geometrically available cell-cell collisions. Finally, we are currently integrating cell aggregation in the free stream with cell adhesion in proximity to the endothelium, and we have proposed a unified framework to describe the cell dynamics within the blood vessel.

In addition, we discussed the single-bond hypothesis in the probabilistic model of small system binding kinetics and concluded that no single criterion is sufficient to support the single bond observation, but a cumulative body of evidence may provide reasonable confidence when a point attachment is assumed to be a quantum unit [85]. We are also developing a non-simplified model of competitive binding of receptor and ligand interactions when at least one type of molecules is both in solution and presented on cell surface, which is 


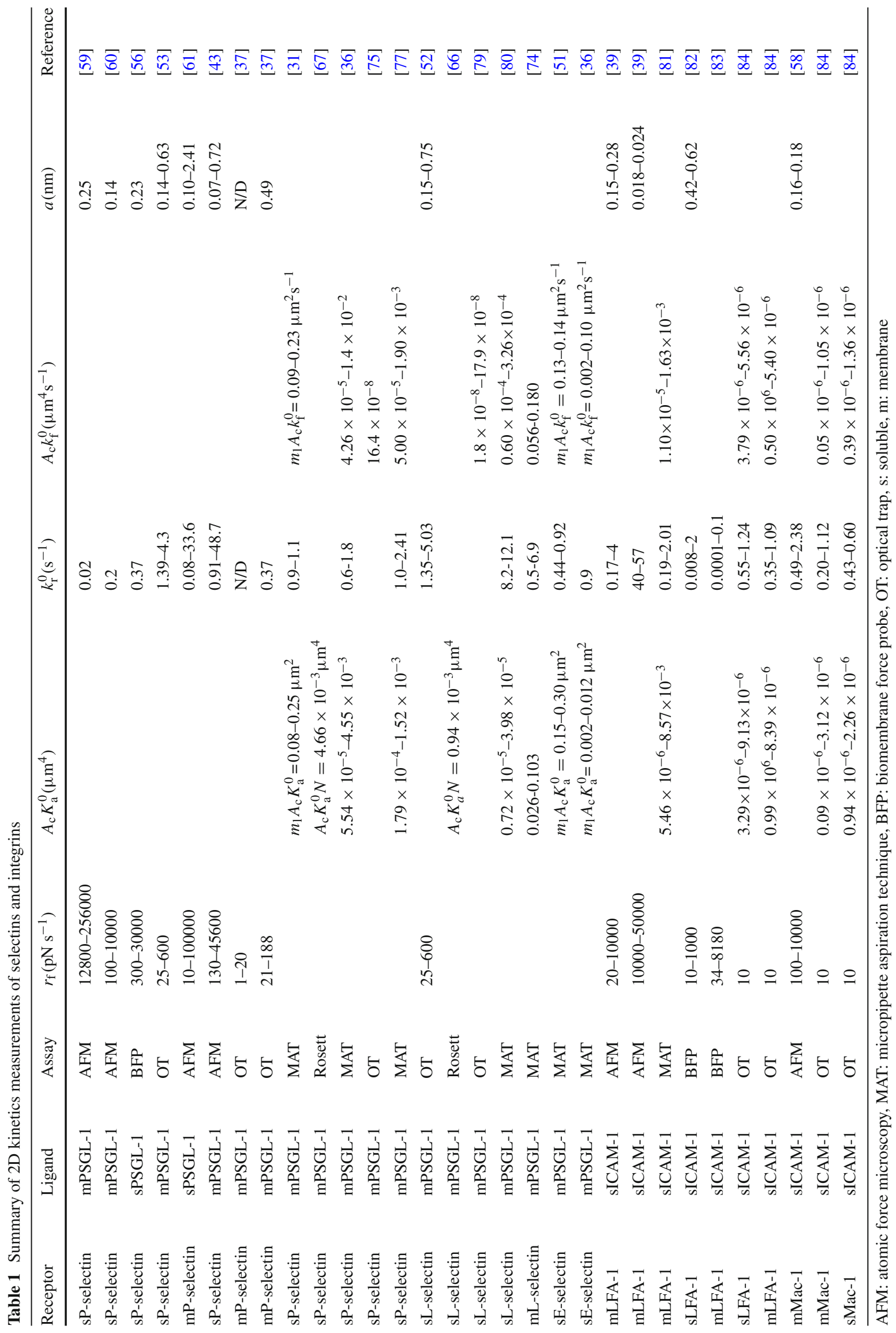


used to quantify the cell adhesion due to the shedding of cellular adhesive molecules off the surface.

\subsection{Binding kinetics of receptor-ligand interactions}

\subsubsection{Selectin-ligand bindings}

$P$-selectin P-selectin is expressed on platelets and activated endothelial cells. Molecular presentation and surface microtopology of the receptors are crucial to their binding kinetics. The randomized orientation or the shortened extension of Pselectin to its ligand- and antibody-binding epitope above the cell membrane lowers the $2 \mathrm{D}$ binding affinity to the PSGL-1 ligand by reducing the forward rates, but not the reverse rates [31]. Meanwhile, stiffening the carrier alone or in cooperation with surface-roughing lowers the 2D affinity of P-selectin-PSGL-1 interactions by reducing the forward rate, but not the reverse rate, whereas softening the carrier and roughing the surface have opposing effects on the $2 \mathrm{D}$ kinetics [36]. In contrast, the soluble antibody binds with a similar 3D affinity to surface-anchored P-selectins or PSGL-1 constructs regardless of their orientation, length, and carrier stiffness and microtopology $[31,36]$. A classic rosetting assay is modified to estimate the binding affinity of P-selectin to PSGL-1 $\left(=4.66 \times 10^{-3} \mu \mathrm{m}^{4}\right)$ by assuming that the size of the rosettes is Poisson distributed [67], which is well consistent with those measured in other biophysical assays. To visualize consecutive binding-unbinding transitions and then to quantify the association kinetics of P-selectin to PSGL-1, a thermal fluctuation assay is developed and a 2D forward rate is estimated as higher for the long construct than the short one [79], as observed Ref. [31]. We find also that bond formation is reduced by enhancing the diffusivity of the selectin-coupled carrier and appears to be temporal historydependent [79].

L-selectin L-selectin is expressed on leukocytes. The binding affinity of L-selectin to PSGL-1 in an equilibrium state is also estimated using a modified rosetting assay $\left(=0.94 \times 10^{-3} \mu \mathrm{m}^{4}\right)$ [67]. IL-8-induced L-selectin shedding reduced the number of membrane-anchored L-selectins and enhanced their affinity to PSGL-1 by greatly lowering its reverse rate, but slightly reducing the forward rate, suggesting that two opposite impacts control the rolling dynamics of activated PMNs: reducing the molecular presentation to enhance the rolling and lowering the kinetic rates to reduce the rolling [74]. At least one reason for such an observation stems from mowing the microvilli and smoothing of the surface of cells by cytokine-mediated L-selectin shedding [74], resulting in enhanced accessibility of residual L-selectin to its ligand [36]. Similar to those performed for P-selectin, the association kinetics of L-selectin and its dependence on carrier diffusivity are also determined using the thermal fluctuation assay, which is robust in determining $2 \mathrm{D}$ associ- ation kinetics and sensitive to the parameters such as sampling rate, sliding window size, and threshold in parametric analysis $[79,86]$.

E-selectin E-selectin is expressed on activated endothelial cells. E-selectin constructs coated on red cells are found to bind in a similar forward rate, but different reverse rate to the ligands expressed on HL-60 or Colo-205 cells bearing carbohydrate ligands, since HL-60 cells only express sLe ${ }^{\mathrm{x}}$, but Colo-205 cells express both $\mathrm{SLe}^{\mathrm{x}}$ and $\mathrm{sLe}^{\mathrm{a}}$ [51]. Again, the randomized orientation of E-selectin to its ligand-binding domain above the cell membrane lowered 2D affinity to the PSGL-1 ligand by reducing the forward rates, but not the reverse rates [31].

Selectin ligands. PSGL-1, serving as a major ligand to Pand L-selectin, presents three sulfated tyrosines and an Oglycan to the interface of the selectin Lec domain. Binding kinetics measurements and molecular dynamics simulations of double or triple tyrosine substitutions indicate that these tyrosines regulate the accessibility of PSGL-1 to P- or Lselectin and that at least one of them is required for PSGL-1 binding to $\mathrm{P}$ - or L-selectin, which results structurally from the significant conformational change of PSGL-1 peptide, but not its binding site of O-glycan [80].

\subsubsection{Integrin-ligand bindings}

Two $\beta_{2}$-integrins members, Mac-1 $\left(\alpha_{M} \beta_{2}\right)$ and LFA-1 $\left(\alpha_{L} \beta_{2}\right)$, are constitutively expressed on PMNs and mediate the PMN recruitment cascade by binding to ICAM-1 expressed on activated endothelial cells under blood flow. Ligand binding of $\beta_{2}$-integrin is regulated by or induces conformational changes in the inserted (I) domain. A surprising discrepancy was revealed between the $2 \mathrm{D}$ and $3 \mathrm{D}$ reverse rate measurements of LFA-1, since 2D reverse rates of the locked open and locked closed I domains only differ a few fold, which is in sharp contrast to the 3D reverse rate of $\sim 100$-fold difference [81]. Even with similar structures, LFA-1 and Mac-1 play distinct roles in PMN recruitment, that is, the slow rolling and firm adhesion of leukocytes rely on LFA-1 while cell crawling is dependent on Mac-1. Such distinct functions are assumed to be governed by the differences in their binding affinities and kinetic rates. The difference in binding affinity between Mac-1 and LFA-1 is forward-rate dominated with a slightly or moderately varied reverse rate. This finding was further confirmed when both $\beta_{2}$-integrins were activated by chemokines (fMLF or IL-8), divalent cations $\left(\mathrm{Mg}^{2+}\right.$ or $\mathrm{Mn}^{2+}$ ), or disulfide bond lockage on an HA state. Structural analyses reveal that such kinetics difference is likely attributed to the distinct conformations at the interface of Mac-1 or LFA-1 and ICAM-1 [87]. PMN spreading is mediated specifically by $\beta_{2}$-integrin-ICAM- 1 interactions and bi-directionally regulated under shear flow. $\alpha_{L}, \alpha_{M}$ and $\beta_{2}$ 
subunits contribute distinctly to PMN spreading on ICAM-1 substrates [88].

Interactions between $\beta_{2}$-integrin on PMNs and ICAM1 on melanoma cells initiate the bindings of melanoma cells to PMNs within the tumor microenvironment in blood flow, which in turn activate PMN-melanoma cell aggregation in a near-wall region of the vascular endothelium, thereby enhancing subsequent extravasation of melanoma cells in the microcirculations. Hydrodynamic shear regulates PMNmelanoma cell heterotypic aggregation that is dependent on shear rates rather than shear stresses. The heterotypic aggregation appears to reach a peak after $\sim 60 \mathrm{~s}$ under shear and then starts to decrease afterward [68]. The cellular binding affinity of a PMN-melanoma cell pair is higher than that of a PMN-endothelial cell pair, but the effective binding affinities per molecular pair are comparable between the two cell pairs no matter whether the melanoma cell or endothelial cell are quiescent or cytokine-activated, indicating that the stronger adhesion between the PMN-melanoma cell pair is mainly attributed to the high expression of ICAM-1 on the melanoma cell. These results propose an alternative mechanism where melanoma cells adhere first with PMNs near vessel wall regions and then bind to endothelial cells via PMNs under blood flow [35].

\subsection{Forced dissociation of receptor-ligand binding}

\subsubsection{Selectin-ligand bonds}

External forces are found to affect the bond of receptorligand interactions. While the lifetime of the P-selectin-G1 $\mathrm{mAb}$ bond is reduced monotonically with applied forces (slip bond), the P-selectin-PSGL-1 bond yields an ascending phase (catch bond) followed by a descending phase with increased force [48]. There are several physical factors to regulate the forced dissociation of the receptor-ligand complex. For instance, the rupture force of P-selectin-PSGL-1 binding reaches a saturated plateau, following a transition phase, with the contact time and yields a threshold with the approach velocity, while the adhesion probability presents a biphasic feature with the retraction velocity [43]. A piecewise linear relationship between rupture force and logarithm of the loading rate is found at high $\left(\sim 10^{4} \mathrm{pN} \mathrm{s}^{-1}\right)$ [43] and low $\left(\sim 10^{-1} \mathrm{pN} \mathrm{s}^{-1}\right)$ [89] for the P-selectin-PSGL-1 bond while the contribution of non-specific binding to the measured rupture force is not negligible at the higher loading rate $\left(>89 \mathrm{pN} \mathrm{s}^{-1}\right)$ [84]. At a low loading rate $\left(<20 \mathrm{pN} \mathrm{s}^{-1}\right)$, rupture force varies with different combinations of spring constant and retraction velocity even at the same loading rate and increases with the spring constant, indicating that the bond dissociation of P-selectin-PSGL-1 interactions is spring constant-dependent at a low loading rate and that the bond rupture force depends on both loading rate and mechan- ical compliance of the force transducer at a high loading rate [37]. Not only the rupture force, but also the bond lifetime is sensitive to the spring constant of the force transducer used. One finds that the bond lifetime of P-selectin-PSGL1 interactions presents stochastic distributions at different spring constants and the catch bond nature is visualized at $\geq 3.0 \times 10^{-2} \mathrm{pN} \mathrm{nm}^{-1}[90]$.

\subsubsection{Integrin-ligand bonds}

Shear resistance of $\beta_{2}$-integrin-ICAM-1 is critical to maintain the slowing rolling, firm adhesion, and crawling of PMNs onto the endothelium under blood flow. A fast and a slow linear loading regime are separately observed in the dynamic force spectra of the LFA-1-ICAM-1 bond, which indicates a steep inner activation barrier and a wide outer activation barrier, respectively. The equilibrium dissociation constant of the LFA-1-ICAM-1 interaction is regulated by the energetics of the outer activation barrier of the complex, while the ability of the complex to resist a pulling force is determined by the divalent cation-dependent inner activation barrier [39]. The binding probability and adhesion force of Mac-1 with ICAM-1 are enhanced upon Mac-1 activation [58]. Small molecule agonist Leukadherin-1 fosters binding of Mac-1 to ICAM-1 via the formation of long membrane tethers, whereas $\mathrm{Mn}^{2+}$ additionally increases ICAM-1 binding via cytoskeleton-anchored bonds [91]. Long lifetimes and increased bond strength also occur when $\beta_{2}$-integrin is activated $[82,83]$. Three states with distinct reverse rates are identified from lifetime distributions of LFA-1-ICAM-1 bond. Force shifts the associated fractions from the short- to intermediate- and long-lived states, producing catch bonds at low forces, but increases their reverse rates exponentially, converting catch to slip bonds at high forces [45]. One also notes that the elasticity of extracellular matrix (ECM) regulates the integrin affinity. For example, a soft ECM increases the activation level of integrins while a stiff ECM has a tendency to prevent the dissociation and internalization of bound integrins $[92,93]$.

\subsection{Structural bases of receptor-ligand interactions}

\subsubsection{Selectin-ligand interactions}

Molecular structure and conformation governs its functions and varies with local chemical and biological environments. For example, applying external forces enable the structural collapse of the P-selectin molecule, which is mainly attributed to the burst of hydrogen bonds within the major $\beta$ sheet of its EGF domain and the disruption of two hydrophobic cores of its Lec domain [94]. The intramolecular extension is also observed in the forced dissociation of P-selectin-PSGL-1 complex prior to the intermolecular sepa- 
ration of fucose group of PSGL-1 from $\mathrm{Ca}^{2+}$ ion of P-selectin [95]. Further allosteric simulations indicate that the bent conformation of EGF-like domain of P-selectin with respect to its Lec domain is unable to switch directly to an extended conformation but presents a spontaneous allostery to a novel, relatively extended conformation starting with the separation between residues Q30 and K67 and terminating with the release of residue N87 from residue C109 [96]. More physiologically, two new molecular dynamics approaches, one involving the shear flow field with a controlled velocity gradient and the other presenting track dragging with a defined trajectory are developed to investigate the microstructural evolution and dissociation kinetics of P-selectin-PSGL-1 interactions under shear flow, indicating that the shear flow alone induces the destruction of Lec/EGF domains within the P-selectin construct before the complex dissociates and that the cooperation of shear flow and tensile stretch mediates the intramolecular destruction of EGF domain and the breaking of hydrogen bond clusters at the P-selectin Lec/EGF interface [97].

\subsubsection{Integrin-ligand interactions}

Molecular dynamics simulations are also employed to elucidate the conformational stability of $\alpha$ subunit I domains of LFA-1 and Mac-1 in different affinity states and relevant I domain-ICAM-1 interaction features. Compared with low LA Mac-1, the LA LFA-1 I domain is unstable in the presence or absence of ICAM-1 ligand, stemming from diverse orientations of its $\alpha 7$-helix with different motifs of a zipper-like, hydrophobic junction between $\alpha 1$ - and $\alpha 7$ helices. Meanwhile, spontaneous transition of the LFA-1 I domain from a LA state to an IA state is first visualized. LA Mac-1 I domain is not favorable for ICAM-1 binding but HA Mac-1 and HA/LA LFA-1 I domain is able to bind to the ICAM-1 ligand readily in free MD simulations, implying that the binding pocket of LA Mac-1 could not open spontaneously, presumably because S144 residue prevents the cation in MIDAS from interacting with D229 residue of ICAM-1 D3 domain [98]. Distinct interface conformations are found between Mac-1 and LFA-1 in different affinity states, which determines the accessibility and availability of MIDAS cation to D3 or D1 domain of ICAM-1 ligand and vary the forward rate value of the two molecules [87].

\section{Summary}

Quantifying 2D kinetics and forced dissociation of receptorligand interactions in cell adhesions is crucial to further the understandings in immune responses. A probabilistic model of a small system is developed to predict $2 \mathrm{D}$ kinetic rates and binding affinities, while a mechanochemical coupling model is introduced to analyze forced regulation of receptorligand interactions. The state-of-the-art techniques including micropipette aspiration and atomic force microscopy are widely used to measure the receptor-ligand binding kinetics and regulation of applied forces. Structural variation, surface environment, and membrane microtopology and stiffness affect the kinetic rates and affinities. Applied forces regulate the bond strength and lifetime in multiple phases.

Acknowledgments This work was supported by Natural Science Foundation of China (grants 10042001, 10072071, 10128205, 30225027, 10332060, 30730032, 11072251, and 31110103918), National Key Basic Research Foundation of China (grants 2006CB910303 and 2011CB710904), National High Technology Research and Development Program of China (grants 2007AA02Z306 and 2011AA020109), Chinese Academy of Sciences (grants KJCX2-L02, KJCX2-SW-L06, 2005-1-16, KJCX2-YW-L08, Y2010030, XDA01030102, XDA04073 801), as well as NIH Fogarty International Research Collaboration Award TW 05774-01. Simulations were conducted on the DeepComp 7000 supercomputer at the Computer Network Information Center, Chinese Academy of Sciences.

\section{References}

1. Zimmerman, G.A., McIntyre, T.M., Prescott, S.M.: Adhesion and signaling in vascular cell-cell interactions. J. Clin. Invest. 98, 1699-1702 (1996)

2. Ginsberg, M.H., Ruggeri, Z.M., Varki, A.P.: Cell adhesion in vascular biology: series introduction. J. Clin. Invest. 98, 1505-1505 (1996)

3. Schmid-Schonbein, G.W.: Analysis of inflammation. Annu. Rev. Biomed. Eng. 8, 93-151 (2006)

4. McEver, R.P., Cummings, R.D.: Role of PSGL-1 binding to selectins in leukocyte recruitment. J. Clin. Invest. 100, 485-492 (1997)

5. Ley, K.: The role of selectins in inflammation and disease. Trends Mol. Med. 9, 263-268 (2003)

6. McEver, R.P.: Selectins. Curr. Opin. Immunol. 6, 75-84 (1994)

7. Li, P., Selvaraj, P., Zhu, C.: Analysis of competition binding between soluble and membrane-bound ligands for cell surface receptors. Biophys. J. 77, 3394-3406 (1999)

8. Chang, K.C., Tees, D.F.J., Hammer, D.A.: The state diagram for cell adhesion under flow: leukocyte rolling and firm adhesion. Proc. Natl. Acad. Sci. USA. 97, 11262-11267 (2000)

9. Snapp, K.R., Craig, R., Herron, M., et al.: Dimerization of Pselectin glycoprotein ligand-1 (PSGL-1) required for optimal recognition of P-selectin. J. Cell Biol. 142, 263-270 (1998)

10. Li, F.G., Erickson, H.P., James, J.A., et al.: Visualization of Pselectin glycoprotein ligand-1 as a highly extended molecule and mapping of protein epitopes for monoclonal antibodies. J. Biol. Chem. 271, 6342-6348 (1996)

11. Hemmerich, S., Leffler, H., Rosen, S.D.: Structure of the $O$-glycans in GlyCAM-1, an endothelial-derived ligand for L-selectin. J. Biol. Chem. 270, 12035-12047 (1995)

12. Ley, K., Laudanna, C., Cybulsky, M.I., et al.: Getting to the site of inflammation: the leukocyte adhesion cascade updated. Nat. Rev. Immunol. 7, 678-689 (2007)

13. Bolomini-Vittori, M., Laudanna, C.: Integrin activation in the immune system. WIREs Syst. Biol. Med. 1, 116-127 (2009) 
14. Shimaoka, M., Takagi, J., Springer, T.A.: Conformational regulation of integrin structure and function. Annu. Rev. Biophys. Biomol. Struct. 31, 485-516 (2002)

15. Arnaout, M.A., Mahalingam, B., Xiong, J.P.: Integrin structure, allostery, and bidirectional signaling. Annu. Rev. Cell Dev. Biol. 21, 381-410 (2005)

16. Luo, B.H., Springer, T.A.: Integrin structures and conformational signaling. Curr. Opin. Cell Biol. 18, 579-586 (2006)

17. Zarbock, A., Kuwano, Y., Spelten, O., et al.: Rolling on E- or Pselectin induces the extended but not high-affinity conformation of LFA-1 in neutrophils. Blood. 116, 617-624 (2014)

18. Kinashi, T.: Intracellular signalling controlling integrin activation in lymphocytes. Nat. Rev. Immunol. 5, 546-559 (2005)

19. Yago, T., Shao, B.J., Miner, J.J., et al.: E-selectin engages PSGL-1 and CD44 through a common signaling pathway to induce integrin alpha(L)beta(2)-mediated slow leukocyte rolling. Blood 116, 485494 (2010)

20. Ginsberg, M.H., Shattil, S.J., Kim, C.: The final steps of integrin activation: the end game. Nat. Rev. Mol. Cell Biol. 11, 288-300 (2010)

21. Diamond, M.S., Staunton, D.E., Marlin, S.D., et al.: Binding of the integrin Mac-1 (CD11b/CD18) to the 3rd immunoglobulin-like domain of ICAM-1 (CD54) and its regulation by glycosylation. Cell 65, 961-971 (1991)

22. Staunton, D.E., Dustin, M.L., Erickson, H.P., et al.: The arrangement of the immunoglobulin-like domains of ICAM-1 and the binding-sites for LFA-1 and rhinovirus. Cell 61, 243-254 (1990)

23. Nagarajan, S., Chesla, S., Cobern, L., et al.: Ligand-binding and phagocytosis by CD16 (Fc-gamma receptor-III) isoformsphagocytic signaling by associated zeta-subunits and gammasubunits in chinese-hamster ovary cells. J. Biol. Chem. 270, 25762-25770 (1995)

24. Nagarajan, S., Venkiteswaran, K., Anderson, M., et al.: Cellspecific, activation-dependent regulation of neutrophil CD32A ligand-binding function. Blood 95, 1069-1077 (2000)

25. Chesla, S.E., Selvaraj, P., Zhu, C.: Measuring two-dimensional receptor-ligand binding kinetics by micropipette. Biophys. J. 75, 1553-1572 (1998)

26. Dembo, M., Torney, D.C., Saxman, K., et al.: The reaction-limited kinetics of membrane-to-surface adhesion and detachment. Proc. R. Soc. Lond. B 234, 55-83 (1988)

27. Bell, G.I.: Models for specific adhesion of cells to cells. Science 200, 618-627 (1978)

28. Tees, D.F.J., Waugh, R.E., Hammer, D.A.: A microcantilever device to assess the effect of force on the lifetime of selectincarbohydrate bonds. Biophys. J. 80, 668-682 (2001)

29. Yuan, C.B., Chen, A., Kolb, P., et al.: Energy landscape of streptavidin-biotin complexes measured by atomic force microscopy. Biochemistry 39, 10219-10223 (2000)

30. Huang, J., Edwards, L.J., Evavold, B.D., et al.: Kinetics of MHCCD8 interaction at the T cell membrane. J. Immunol. 179, 76537662 (2007)

31. Huang, J., Chen, J., Chesla, S.E., et al.: Quantifying the effects of molecular orientation and length on two-dimensional receptorligand binding kinetics. J. Biol. Chem. 279, 44915-44923 (2004)

32. McQuarrie, D.A.: Kinetics of small systems.1. J. Chem. Phys. 38, 433-436 (1963)

33. Zhu, C., Bao, G., Wang, N.: Cell mechanics: mechanical response, cell adhesion, and molecular deformation. Annu. Rev. Biomed. Eng. 2, 189-226 (2000)

34. Zhu, C.: Kinetics and mechanics of cell adhesion. J. Biomech. 33, 23-33 (2000)

35. Fu, C.L., Tong, C.F., Wang, M.L., et al.: Determining beta(2)integrin and intercellular adhesion molecule 1 binding kinetics in tumor cell adhesion to leukocytes and endothelial cells by a gas-driven micropipette assay. J. Biol. Chem. 286, 34777-34787 (2011)

36. Wu, L., Xiao, B.T., Jia, X.L., et al.: Impact of carrier stiffness and microtopology on two-dimensional kinetics of P-selectin and P-selectin glycoprotein ligand-1 (PSGL-1) interactions. J. Biol. Chem. 282, 9846-9854 (2007)

37. Zhang, Y., Sun, G.Y., Lü, S.Q., et al.: Low spring constant regulates P-selectin-PSGL-1 bond rupture. Biophys. J. 95, 5439-5448 (2008)

38. Wojcikiewicz, E.P., Abdulreda, M.H., Zhang, X.H., et al.: Force spectroscopy of LFA-1 and its ligands, ICAM-1 and ICAM-2. Biomacromolecules 7, 3188-3195 (2006)

39. Zhang, X.H., Wojcikiewicz, E., Moy, V.T.: Force spectroscopy of the leukocyte function-associated antigen-1/intercellular adhesion molecule-1 interaction. Biophys. J. 83, 2270-2279 (2002)

40. Hukkanen, E.J., Wieland, J.A., Gewirth, A., et al.: Multiple-bond kinetics from single-molecule pulling experiments: evidence for multiple NCAM bonds. Biophys. J. 89, 3434-3445 (2005)

41. Evans, E.: Probing the relation between force-lifetime-and chemistry in single molecular bonds. Annu. Rev. Biophys. Biomol. Struct. 30, 105-128 (2001)

42. Bonanni, B., Kamruzzahan, A.S.M., Bizzarri, A.R., et al.: Single molecule recognition between cytochrome C 551 and goldimmobilized Azurin by force spectroscopy. Biophys. J. 89, 27832791 (2005)

43. Lü, S.Q., Ye, Z.Y., Zhu, C., et al.: Quantifying the effects of contact duration, loading rate, and approach velocity on Pselectin-PSGL-1 interactions using AFM. Polymer 47, 2539-2547 (2006)

44. Kong, F., Garcia, A.J., Mould, A.P., et al.: Demonstration of catch bonds between an integrin and its ligand. J. Cell Biol. 185, 12751284 (2009)

45. Chen, W., Lou, J.Z., Zhu, C.: Forcing switch from short- to intermediate- and long-lived states of the alpha A domain generates LFA-1/ICAM-1 catch bonds. J. Biol. Chem. 285, 35967-35978 (2010)

46. Lou, J.Z., Yago, T., Klopocki, A.G., et al.: Flow-enhanced adhesion regulated by a selectin interdomain hinge. J. Cell Biol. 174, 1107 1117 (2006)

47. McEver, R.P., Zhu, C.: Rolling cell adhesion. Annu. Rev. Cell Dev. Biol. 26, 363-396 (2010)

48. Marshall, B.T., Long, M., Piper, J.W., et al.: Direct observation of catch bonds involving cell-adhesion molecules. Nature 423, 190$193(2003)$

49. Wu, Y., Vendomea, J., Shapiroa, L., et al.: Transforming binding affinities from $3 \mathrm{D}$ to $2 \mathrm{D}$ with application to cadherin clustering. Nature 475, 510-513 (2011)

50. Kaplanski, G., Farnarier, C., Tissot, O., et al.: Granulocyte endothelium initial adhesion: analysis of transient binding events mediated by E-selectin in a laminar shear-flow. Biophys. J. 64, 1922-1933 (1993)

51. Long, M., Zhao, H., Huang, K.S., et al.: Kinetic measurements of cell surface E-selectin/carbohydrate ligand interactions. Ann. Biomed. Eng. 29, 935-946 (2001)

52. Svoboda, K., Schmidt, C.F., Schnapp, B.J., et al.: Direct observation of kinesin stepping by optical trapping interferometry. Nature 365, 721-727 (1993)

53. Rinko, L.J., Lawrence, M.B., Guilford, W.H.: The molecular mechanics of P- and L-selectin lectin domains binding to PSGL-1. Biophys. J. 86, 544-554 (2004)

54. Husson, J., Chemin, K., Bohineust, A., et al.: Force generation upon T cell receptor engagement. Plos One 6, e19680 (2011)

55. Axmann, M., Huppa, J.B., Davis, M.M., et al.: Determination of interaction kinetics between the $\mathrm{T}$ cell receptor and peptide-loaded MHC class II via single-molecule diffusion measurements. Biophys. J. 103, L17-L19 (2012) 
56. Evans, E., Leung, A., Heinrich, V., et al.: Mechanical switching and coupling between two dissociation pathways in a P-selectin adhesion bond. Proc. Natl. Acad. Sci. 101, 11281-11286 (2004)

57. Merkel, R., Nassoy, P., Leung, A., et al.: Energy landscapes of receptor-ligand bonds explored with dynamic force spectroscopy. Nature 397, 50-53 (1999)

58. Yang, H.Y., Yu, J.P., Fu, G., et al.: Interaction between single molecules of Mac-1 and ICAM-1 in living cells: an atomic force microscopy study. Exp. Cell Res. 313, 3497-3504 (2007)

59. Fritz, J., Katopodis, A.G., Kolbinger, F., et al.: Force-mediated kinetics of single P-selectin ligand complexes observed by atomic force microscopy. Proc. Natl. Acad. Sci. 95, 12283-12288 (1998)

60. Hanley, W., McCarty, O., Jadhav, S., et al.: Single molecule characterization of P-selectin/ligand binding. J. Biol. Chem. 278, 10556-10561 (2003)

61. Marshall, B.T., Sarangapani, K.K., Lou, J.H., et al.: Force history dependence of receptor-ligand dissociation. Biophys. J. 88, 14581466 (2005)

62. Alon, R., Chen, S.Q., Puri, K.D., et al.: The kinetics of L-selectin tethers and the mechanics of selectin-mediated rolling. J. Cell. Biol. 138, 1169-1180 (1997)

63. Kitayama, J., Fuhlbrigge, R.C., Puri, K.D., et al.: P-selectin, Lselectin, and alpha(4) integrin have distinct roles in eosinophil tethering and arrest on vascular endothelial cells under physiological flow conditions. J. Immunol. 159, 3929-3939 (1997)

64. Schmidtke, D.W., Diamond, S.L.: Direct observation of membrane tethers formed during neutrophil attachment to platelets or P-selectin under physiological flow. J. Cell Biol. 149, 719-729 (2000)

65. Finger, E.B., Puri, K.D., Alon, R., et al.: Adhesion through Lselectin requires a threshold hydrodynamic shear. Nature 379, 266269 (1996)

66. Riper, J.W., Swerlick, R.A., Zhu, C.: Determining force dependence of two-dimensional receptor-ligand binding affinity by centrifugation. Biophys. J. 74, 492-513 (1998)

67. Long, M., Chen, J., Jiang, N., et al.: Probabilistic modeling of rosette formation. Biophys. J. 91, 352-363 (2006)

68. Liang, S.L., Fu, C.L., Wagner, D., et al.: Two-dimensional kinetics of beta(2)-integrin and ICAM-1 bindings between neutrophils and melanoma cells in a shear flow. Am. J. Physiol. 294, C743-C753 (2008)

69. Long, M., Goldsmith, H.L., Tees, D.F.J., et al.: Probabilistic modeling of shear-induced formation and breakage of doublets cross-linked by receptor-ligand bonds. Biophys. J. 76, 1112-1128 (1999)

70. Bayas, M.V., Kearney, A., Avramovic, A., et al.: Impact of salt bridges on the equilibrium binding and adhesion of human CD2 and CD58. J. Biol. Chem. 282, 5589-5596 (2007)

71. Leckband, D.: Design rules for biomolecular adhesion: lessons from force measurements. In: Prausnitz, J.M., Doherty, M.F., Segalman, R.A. (eds.) Annual Review of Chemical and Biomolecular Engineering, vol. 1, pp. 365-389. Annual Reviews, Palo Alto (2010)

72. Tolentino, T.P., Wu, J.H., Zarnitsyna, V.I., et al.: Measuring diffusion and binding kinetics by contact area FRAP. Biophys. J. 95, 920-930 (2008)

73. Wu, J.H., Fang, Y., Zarnitsyna, V.I., et al.: A coupled diffusionkinetics model for analysis of contact-area FRAP experiment. Biophys. J. 95, 910-919 (2008)

74. Jia, X.L., Chen, J., Long, M.: IL-8-induced L-selectin shedding regulates its binding kinetics to PSGL-1. Chin. Sci. Bull. 54, 27862793 (2009)

75. Huppa, J.B., Axmann, M., Mörtelmaier, M.A., et al.: TCRpeptide-MHC interactions in situ show accelerated kinetics and increased affinity. Nature 463, 963-967 (2010)
76. Grashoff, C., Hoffman, B.D., Brenner, M.D., et al.: Measuring mechanical tension across vinculin reveals regulation of focal adhesion dynamics. Nature 466, 263-266 (2010)

77. Chakraborty, S., Núñez, D., Hu, S., et al.: FRET based quantification and screening technology platform for the interactions of leukocyte function-associated antigen-1 (LFA-1) with intercellular adhesion molecule-1 (ICAM-1). Plos One 9, e102572 (2014)

78. Chigaev, A., Buranda, T., Dwyer, D.C., et al.: FRET detection of cellular $\alpha 4$-integrin conformational activation. Biophys. J. 85, 3951-3962 (2003)

79. Sun, G.Y., Zhang, Y., Huo, B., et al.: Surface-bound selectin-ligand binding is regulated by carrier diffusion. Eur. Biophys. J. 38, 701$711(2009)$

80. Xiao, B.T., Tong, C.F., Jia, X.L., et al.: Tyrosine replacement of PSGL-1 reduces association kinetics with P- and L-selectin on the cell membrane. Biophys. J. 103, 777-785 (2012)

81. Zhang, F., Marcus, W.D., Goyal, N.H., et al.: Two-dimensional kinetics regulation of alpha(L)beta(2)-ICAM-1 interaction by conformational changes of the alpha(L)-inserted domain. J. Biol. Chem. 280, 42207-42218 (2005)

82. Evans, E., Kinoshita, K., Simon, S., et al.: Long-lived, high-strength states of ICAM-1 bonds to beta(2) integrin, I: lifetimes of bonds to recombinant alpha(L) beta(2) under force. Biophys. J. 98, 14581466 (2010)

83. Kinoshita, K., Leung, A., Simon, S., et al.: Long-lived, highstrength states of ICAM-1 bonds to beta(2) integrin, II: lifetimes of LFA-1 bonds under force in leukocyte signaling. Biophys. J. 98, 1467-1475 (2010)

84. Li, N., Zhang, Y., Sun, G.Y., et al.: Rupture force and lifetime measurements for non-specific interactions. J. Med. Biomech. 22, 35-39 (2007)

85. Zhu, C., Long, M., Chesla, S.E., et al.: Measuring receptor/ligand interaction at the single-bond level: experimental and interpretative issues. Ann. Biomed. Eng. 30, 305-314 (2002)

86. Sun, G.Y., Zhang, Y., Huo, B., et al.: Parametric analysis for monitoring 2D kinetics of receptor-ligand binding. Cell. Mole. Bioeng. 2, 495-503 (2009)

87. Li, N., Mao, D.B., Lü, S.Q., et al.: Distinct binding affinities of Mac-1 and LFA-1 in neutrophil activation. J. Immunol. 190, 4371$4381(2013)$

88. Zhan, D.Y., Zhang, Y., Long, M.: Spreading of human neutrophils on an ICAM-1-immobilized substrate under shear flow. Chin. Sci. Bull. 57, 769-775 (2012)

89. Zhang, Y., Ye, Z.Y., Huo, B., et al.: Rupture force measurements for P-selectin/PSGL-1 bonds using an optical trap assay. J. Med. Biomech. 22, 961-965 (2005)

90. Zhang, Y., Lü, S.Q., Long, M.: Probe stiffness regulates receptorligand bond lifetime under force. Sci. Chin. 54, 923-929 (2011)

91. Celik, E., Faridi, M.H., Kumar, V., et al.: Agonist Leukadherin-1 increases CD11b/CD18-dependent adhesion via membrane tethers. Biophys. J. 105, 2517-2527 (2013)

92. Xu, G., Feng, X., Zhao, H., et al.: Theoretical study of the competition between cell-cell and cell-matrix adhesions. Phys. Rev. E. 80, 011921 (2009)

93. Xu, G., Yang, C., Du, J., et al.: Integrin activation and internalization mediated by extracellular matrix elasticity: a biomechanical model. Biomechanics 47, 1479-1484 (2014)

94. Lü, S.Q., Long, M.: Forced extension of P-selectin construct using steered molecular dynamics. Chin. Sci. Bull. 49, 10-17 (2004)

95. Lü, S., Long, M.: Forced dissociation of selectin-ligand complexes using steered molecular dynamics simulation. Mol. Cell. Biomech. 2, 161-177 (2005)

96. Lü, S.Q., Zhang, Y., Long, M.: Visualization of allostery in Pselectin lectin domain using MD simulations. Plos One 5, e15417 (2010) 
97. Kang, Y.Y., Lü, S.Q., Ren, P., et al.: Molecular dynamics simulation of shear- and stretch-induced dissociation of P-selectin/PSGL-1 complex. Biophys. J. 102, 112-120(2012)
98. Mao, D.B., Lü, S.Q., Li, N., et al.: Conformational stability analyses of alpha subunit I domain of LFA-1 and Mac-1. Plos One 6, e24188 (2011) 